\title{
Efeito modulador da interface EMG-FES na abertura de mão em indivíduos após AVC: Estudo de casos
}

\author{
EMG-FES interface modulating effect during open hand \\ movement post stroke: Case reports
}

\begin{abstract}
Efecto de la interfaz EMG-FES en la apertura de la mano en personas después del accidente cerebrovascular: Estudio de casos
\end{abstract}

\author{
Giovanna Luise Cabrera Fumagali ${ }^{1}$, Eddy Krueger $^{2}$
}

\begin{abstract}
1.Acadêmica de Fisioterapia, Departamento de Fisioterapia, Laboratório de Engenharia Neural e de Reabilitação, UEL. Londrina-PR, Brasil. https://orcid.org/0000-0002-6155-044X

2.Docente na Universidade Estadual de Londrina - UEL. Departamento de Anatomia, Laboratório de Engenharia Neural e de Reabilitação. Londrina-PR, Brasil. https://orcid.org/0000-0003-3890-1595
\end{abstract}

\section{Resumo}

Objetivo. A eletromiografia (EMG) capta ativação do motoneurônio e aciona a estimulação elétrica funcional (FES), mimetizando a ativação neuromuscular e promovendo a neuroplasticidade. A interface EMG-FES é a interação de ambos os dispositivos, seu uso no presente estudo de casos tem como objetivo verificar seu efeito modulador na abertura da mão em participantes pós-acidente vascular cerebral (AVC). Método. Foram realizadas 4 avaliações: (1) antes da intervenção, (2) após 8 sessões, (3) após 16 sessões e (4) acompanhamento após 15 dias da última intervenção. Foram feitas EMG de superfície nos músculos: (i) extensores de dedos, (ii) extensor do indicador, (iii) abdutor do polegar e (iv) flexor dos dedos. Foram realizadas 8 ou 16 sessões de 30 minutos de duração de aplicação da interface EMG-FES nos músculos extensores de dedos. Resultados. Foram contatados 431 e selecionados 4 participantes para o protocolo. Em dois dos participantes foram realizadas 8 sessões e nos outros dois, 16. A EMG registrou aumento do sinal e a taxa de disparo neural dos músculos avaliados na segunda e terceira avaliação, respectivamente. Nas avaliações seguintes houve diminuição dos valores, exceto nos músculos flexores dos dedos. Conclusão. A interface EMG-FES indicou que funciona para aumentar a ativação muscular e a taxa de disparo neural. Na avaliação de acompanhamento houve regressão dos valores, indicando melhora apenas a curto prazo. Para otimização da aplicação da interface EMG-FES sugere-se associá-la à exercícios resistidos e/ou terapia espelho.

Unitermos. Acidente Vascular Cerebral; eletromiografia; estimulação elétrica funcional; reabilitação

\footnotetext{
Abstract

Objective. Electromyography (EMG) captures motor neuron activation and this signal trigger the functional electrical stimulation (FES) that mimics muscle activation and promotes neuroplasticity. The EMG-FES interface is interaction of both devices, its use in the present case reposts aims to verify its modulating effect in improving the hand opening movement in post-stroke participants. Method. The assessments performed: (1) before the intervention, (2) after 8 sessions, (3) after 16 sessions and (4) follow-up after 15 days of the last intervention. EMG assessments were performed on muscles: (i) finger extensors, (ii) index extensor, (iii) thumb abductor and (iv) wrist flexor. The EMG-FES interface was applied on finger extensor muscles by 8 or 16 sessions during about 30 minutes. Results. For application, 431 subjects were contacted, and 4 participants were selected. Were applied $8(\mathrm{~N}=2)$ and 16 $(\mathrm{N}=2)$ sessions. The EMG outcomes indicated increase in muscle activation and neural firing rate in second and third assessments. In the follow up assessment the values decreased,
} 
except for flexor muscles. Conclusion. The EMG-FES interface showed an increase in muscle activation and neural firing rate. The follow-up evaluation did not show improvement. In order to improve EMG-FES interface application, it is suggested to associate resistance exercises and/or mirror therapy.

Keywords. Stroke; Electromyography; Functional electrical stimulation; Rehabilitation

\section{Resumen}

Objetivo. La electromiografía (EMG) captura la activación de la neurona motora y desencadena la estimulación eléctrica funcional (FES) que imita la activación muscular y promueve la neuroplasticidad. La interfaz EMG-FES es la interacción de ambos dispositivos, su uso en el presente estudio tiene como objetivo verificar su efecto modulador en la mejora de la apertura de la mano en los participantes después del accidente cerebrovascular. Método. Se realizaron 4 evaluaciones: (1) antes de la intervención, (2) después de 8 sesiones, (3) después de 16 sesiones y (4) después de 15 días de la última intervención. La EMG de superficie se realizó en los músculos: (i) extensores de los dedos, (ii) extensor índice, (iii) abductor del pulgar y (iv) flexor de los dedos. Realizaron 8 o 16 sesiones de 30 minutos de aplicación de la interfaz EMG-FES en los músculos extensores de los dedos. Resultados. Se contactaron 431 y 4 participantes fueron seleccionados para el protocolo. En dos de los participantes, se realizaron 8 sesiones y en las otras dos, 16. El EMG registró un aumento en la señal y la tasa de activación neuronal de los músculos evaluados en la segunda y tercera evaluaciones, respectivamente. En las siguientes evaluaciones hubo una disminución en los valores, excepto en los músculos flexores de los dedos. Conclusiones. La interfaz EMG-FES indicó un aumento en la activación muscular y la tasa de activación neural. La evaluación de seguimiento mostró que no hubo mejoría. Para optimizar la aplicación de la interfaz EMG-FES, se sugiere asociarla con ejercicios de resistencia y / o terapia de espejo.

Palabras clave. Accidente Cerebrovascular; electromiografía; estimulación eléctrica funcional; rehabilitación

Trabalho realizado na Universidade Estadual de Londrina, Londrina-PR, Brasil.

\section{INTRODUÇÃO}

Para a Organização Mundial da Saúde (OMS), o acidente vascular cerebral (AVC) é definido como uma disfunção neurológica de origem vascular isquêmica ou hemorrágica de início súbito. Tal alteração vascular pode levar a danos teciduais que apresentaram sintomas correspondentes a área cerebral comprometida ${ }^{1}$. O AVC isquêmico é caracterizado pela obstrução de uma ou mais artérias, causada, por exemplo, (i) aterosclerose, (ii) êmbolo ou (iii) 
trombo, levando a falta de oxigenação e morte tecidual, além disso, é comum a presença de edema que também leva a morte de neurônios. O AVC hemorrágico caracterizado pelo sangramento cerebral provocado pelo rompimento de uma artéria, causada, por exemplo, por (i) hipertensão arterial e (ii) problemas na coagulação, dessa forma, o extravasamento de sangue leva a morte do neurônio pela sua toxicidade sanguínea ${ }^{1}$. Quando comprometido a área do cérebro responsável pela motricidade, ocorrem alterações motoras como (i) déficit voluntário, (ii) espasticidade na mão, levando a (iii) incapacidade funcional, principalmente por afetar movimentos manuais ${ }^{2}$.

A eletromiografia (EMG) é um método não invasivo que faz a representação gráfica da atividade elétrica do motoneurônio captado na superfície da pele do músculo que se almeja avaliar. Esse sinal pode ser usado como mecanismo de biofeedback ou para acionar um dispositivo ${ }^{3}$ como, por exemplo, uma prótese robótica.

A estimulação elétrica funcional (FES) objetiva produzir uma função corpórea envolvendo, assim, parâmetros estimulatórios, local de aplicação e tarefa a ser realizada. A corrente da FES necessária para ativar um musculo é inversamente proporcional ao grau de inervação e hipotrofia4. Desse modo, a FES permite ativar grupos musculares com perda de função motora, afetados por alterações neurológicas 5 .

A interface EMG-FES é conduzida por um software que detecta a abertura da mão pela EMG e ativa a FES, de modo 
mimetizar a ativação muscular a partir da intenção de movimento voluntária do usuário, promovendo a neuroplasticidade ${ }^{6}$, a fim de melhorar a função motora e sensitiva da mão 5 . A interface EMG-FES define o limiar do sinal de contração de cada participante para que ele ative a EMG se realmente produzir um movimento voluntário ${ }^{6}$.

$\mathrm{Na}$ reabilitação física convencional das sequelas oriundas do AVC, muitas vezes, as últimas regiões do corpo que alcançam melhoria são as distais, como mão e pé. Um dos fatores positivos na introdução da interface EMG-FES na reabilitação física é buscar melhoria distal antecipadamente ${ }^{7}$. Tendo em vista essa lacuna, o objetivo desta pesquisa foi realizar uma série de estudo de casos $(\mathrm{N}=4)$ para avaliar a possível efetividade da interface músculo-máquina (EMGFES) na melhora do movimento de abertura da mão e dos dedos em participantes com sequela motora em mão oriunda do AVC. Como hipótese, espera-se que a interface aumentará a ativação muscular e taxa de disparo neural mesmo após quinze dias do término das sessões.

\section{MÉTODO}

\section{Amostra}

O projeto foi executado no Laboratório de Engenharia Neural e de Reabilitação (LENeR) localizado nas dependências do departamento de anatomia da Universidade Estadual de Londrina. Este projeto teve aprovação pelo 
Comitê de Ética em Pesquisa envolvendo Seres Humanos (pelo no 3.004.069) da Universidade Estadual de Londrina.

Foram incluídos no estudo indivíduos maiores de 18 anos com sequelas neurológicas pós-AVC com mais de seis meses pós lesão, com sequela caracterizada pela postura de decorticação com predomínio de padrão flexor e paresia em membro superior do hemicorpo afetado, com contração dos extensores de punhos registrado pela EMG, sem contraturas musculares. A avaliação de tônus não foi elencada como critério de inclusão. Além disso, os participantes (ou responsáveis) assinaram o termo de consentimento livre esclarecido.

Foram excluídos indivíduos com plegia de membro superior; alterações dermatológicas que impedissem a captação do sinal de EMG como dermatites; não tolerar o desconforto proporcionado pela FES; presença de tecido neoplásico próximo à área estimulada eletricamente; implante metálico em regiões próximas ou entre os eletrodos; alteração cognitiva que prejudicasse a realização do protocolo e/ou presença de outros distúrbios neurológicos.

\section{Procedimento}

Para a seleção dos participantes foram feitas ligações para contatos fornecidos pelo hospital universitário, estes foram questionados se sofreram AVC e a quanto tempo foi o episódio; se teriam interesse em participar; se possuíam déficit em mão, cognitivo ou incapacidade de sair de casa e 
idade. Após a realização do questionamento, se o indivíduo fosse elegível a participar, era marcada a avaliação presencial em que se avaliava a presença de déficit motor e a paralisia do grupo muscular extensor de punho e dos dedos da mão pela EMG.

\section{Avaliação eletromiográfica}

Foram realizadas avaliações da eletromiografia dos músculos (i) extensores de dedos, (ii) extensor do indicador, (iii) abdutor do polegar e (iv) flexor de punho. $O$ equipamento utilizado para a aquisição foi o Bitalino ${ }^{\circledR}$, modelo MuscleBit, com registro do sinal pelo programa OpenSignals ${ }^{\circledR}$ e OpenVibe ${ }^{\circledR}$.

Para a aquisição, a amplificação do sinal foi de 2000x e a frequência de amostragem foi de $1 \mathrm{kHz}$. Cada canal constituía dois eletrodos (marca $3 \mathrm{M}^{\circledR}$ ) de superfície ( $\mathrm{Ag} / \mathrm{Ag}$ $\mathrm{Cl})$ posicionados sobre o terço médio-distal dos músculos extensor que foram avaliados com um eletrodo de referência no epicôndilo lateral do úmero.

O participante realizou 5 segundos de contração máxima voluntária (sem resistência) por meio do comando verbal "abra sua mão" com 3 repetições. O movimento de abertura de mão foi realizado de maneira espelhada pelo instrutor que se orientou por uma rotina customizada desenvolvida no programa OpenVibe ${ }^{\circledR}$. $O$ intervalo entre as repetições foi randomizado entre 2 a 10 s pelo software para que o participante não tentasse "adivinhar" quando deveria contrair. 
Foi realizada uma avaliação antes da primeira sessão (Pré). Foram realizadas 8 sessões com média de 30 minutos de duração em 2 dos participantes. Após as 8 sessões houve uma reavaliação (Pós8). Foram aplicadas mais 8 sessões e uma outra avaliação (Pós 16 ). Após 15 dias da avaliação Pós ${ }_{16}$, foi feita uma avaliação de acompanhamento (PósFu).

A avaliação do sinal foi realizada por meio de uma rotina customizada pelo programa MatLab ${ }^{\circledR}$. O processamento consistia em filtragem Butterworth de quarta ordem, com passa-banda $(10-450 \mathrm{~Hz})$ e rejeita-faixa em $60 \mathrm{~Hz}$ e harmônicas (120, 180, 240, 300, 360 e 420 Hz). Foram extraídos os descritores valor eficaz (RMS) e frequência mediana (MDF), do domínio do tempo e da frequência, respectivamente.

Aplicação da interface

Após os participantes passarem pela avaliação, era aplicada a interface EMG-FES, a qual foi construída no laboratório. Para a EMG, foi posicionado um canal (dois eletrodos de $\mathrm{Ag} / \mathrm{Ag}-\mathrm{Cl}$ ) no músculo extensor dos dedos, com eletrodo de referência no epicôndilo lateral do úmero, entre os eletrodos bipolares ( $5 \times 5 \mathrm{~cm}$, auto-adesivos, Valutrode ${ }^{\circledR}$ ) da FES como mostra a Figura 1.

Após a colocação dos eletrodos, o programa OpenVibe ${ }^{\circledR}$ foi utilizado para realizar as etapas da intervenção. A primeira etapa consistia em solicitar que o participante realizasse as contrações da mesma forma como ocorreu na avaliação. 
(A)

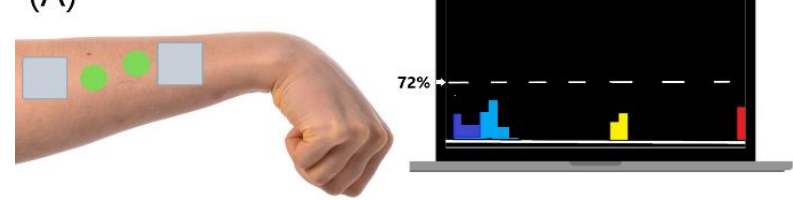

(B)

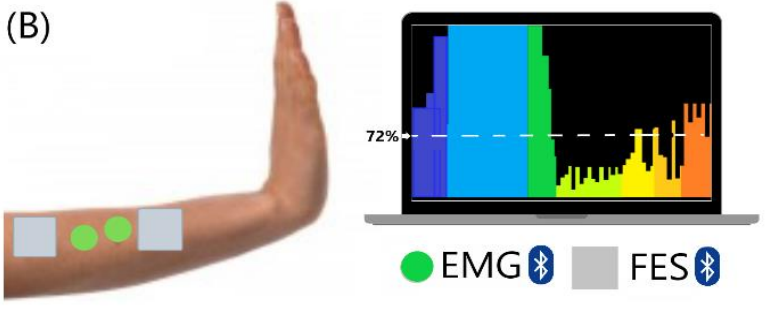

Figura 1. Aplicação da interface EMG-FES: (A) participante em repouso, sinal elétrico registrado pela EMG, é basal sendo assim baixo, (B) participante inicia o movimento até o sinal elétrico registrado pela EMG atinge $72 \%$ para ativar FES. O bluetooth permitia a transmissão do sinal da EMG para o computador e o sinal do computador para a FES.

Após a realização das 3 contrações 0 sinal foi processado como na avaliação e extraído apenas o descritor RMS, onde a média das contrações foi computada. A fim de trabalhar em cima da capacidade residual de cada participante, na segunda etapa, o valor da EMG foi normalizado em cada intervenção, conforme mostra a equação:

$$
R M S_{\text {NORM }}=\frac{R M S_{x}-R M S_{\text {repouso }}}{R M S_{C V M}-R M S_{\text {repouso }}} \cdot 100
$$

Onde, NORM é o valor normalizado, $x$ é o valor instantâneo, repouso é o valor de repouso e CVM é o valor de contração voluntária máxima. Para a terceira etapa, que consistia no treinamento, o participante deveria realizar uma contração que alcançasse $72 \%$ para que a FES fosse ativada como ilustra a Figura 1. 
A FES utilizada é um dispositivo portátil com duas baterias de $12 \mathrm{v}$, com formato de onda bifásico e amplificado por meio de dois eletrodos $5 \times 5 \mathrm{~cm}$ auto-adesivos. Programada com (i) frequência modulada em até $30 \mathrm{~Hz}$, (ii) frequência de portadora em $1 \mathrm{kHz}$, (iii) duração de pulso 400 $\mu$ s (tempo ativo, $200 \mu s$ de fase positiva e $200 \mu s$ de fase negativa), (iv) intensidade ajustada individualmente em nível submáximo. Quanto a potência, o dispositivo de malha aberta têm potência máxima de $24 \mathrm{v}$, possibilitando modulação manual quando notado alterações do padrão muscular ao longo do tempo como a fadiga.

Quando FES era ativada, a aquisição da EMG era interrompida para evitar a interferência entre os dispositivos. O início do movimento tinha frequência aumentava até atingir $30 \mathrm{~Hz}$ de modo alcançar uma extensão quase máxima da mão e mantida por aproximadamente 3 segundos.

Como demonstrada na Figura 2 a ativação pode ser descrita em 4 fases: (1) fase de subida em que o início do movimento a aciona a FES por meio da identificação do movimento pela EMG, a frequência aumentava de $5 \mathrm{~Hz}$ até $30 \mathrm{~Hz}$; (2) fase de manutenção em que atingindo extensão submáxima por aproximadamente 3s; (3) fase de descida em que desativa-a gradualmente, diminuição da frequência de $30 \mathrm{~Hz}$ até $5 \mathrm{~Hz}$; (4) fase desligada em que não há aplicação com 4 segundos de intervalo para poder reativar o sistema. 


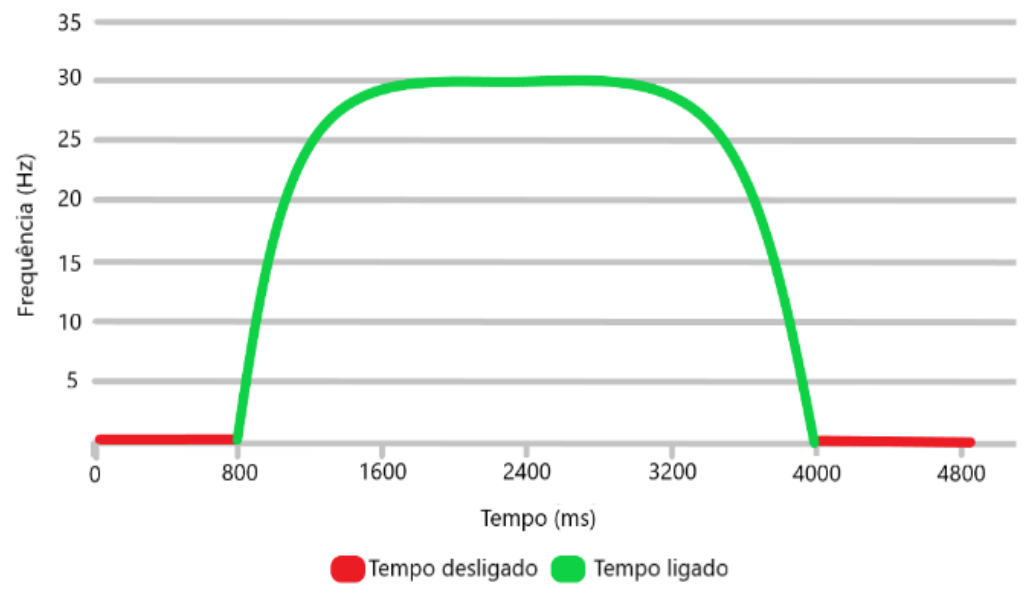

Figura 2. Ilustração da modulação da ativação da FES por meio da variação da frequência e suas 4 fases: subida, manutenção, descida e desligada.

\section{Análise de dados}

A análise dos resultados foi baseada na avaliação EMG dos músculos (i) extensor dos dedos, (ii) extensor do indicador, (iii) abdutor longo do polegar, e (iv) flexor superficial dos dedos dos quatro participantes. Os dados coletados foram organizados em gráficos boxplot, de modo a facilitar a comparação e o interpretação dos dados. Inferência estatística sobre a população não foram executadas pois a relação entre tamanha da população e tamanho da amostra não se mostrou satisfatória para tais conclusões, a amostra Pós 8 contém apenas 4 participantes e

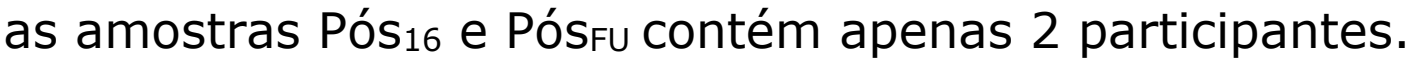

\section{RESULTADOS E DISCUSSÃO}

A Figura 3 mostra que foram contatados 431 possíveis participantes, onde por meio de ligação telefônica, com questionário simples como tempo de lesão, comorbidades, 
dentre outros, foram selecionados 30 indivíduos. Foram marcadas avaliações presenciais para os 30 selecionados, destes 16 não compareceram, 5 não tinham déficit motor e 2 tinham paralisia total de extensores de mão e dedos, dessa forma foram incluídos 7 participantes que estariam aptos a intervenção. Após o início das intervenções 3 desistiram, dessa forma restaram 4 participantes para aplicação do protocolo.

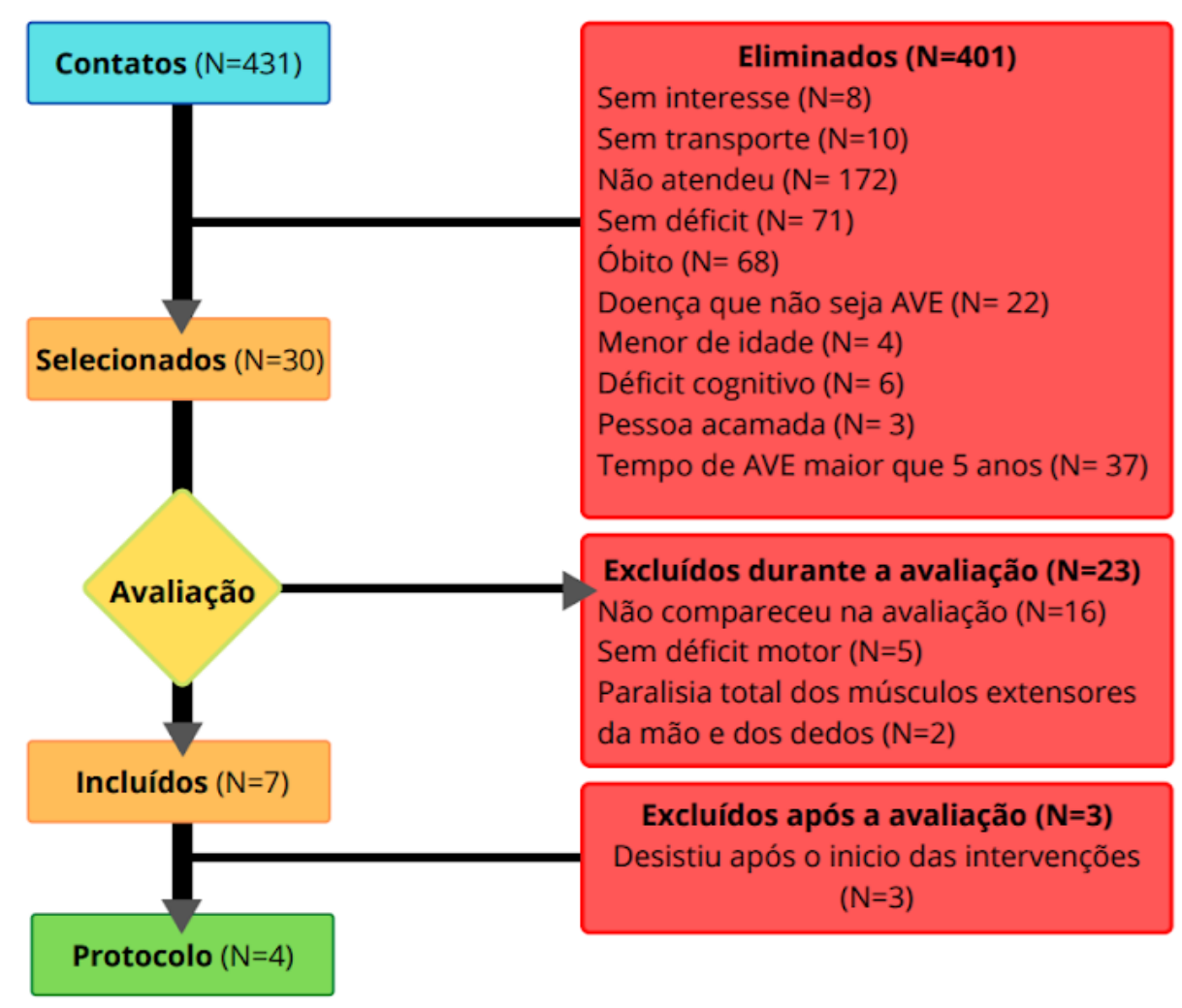

Figura 3. Organograma de seleção dos participantes do estudo, adequado aos critérios de inclusão, com o total de contatos e total de indivíduos que finalizaram o protocolo.

Foi aplicado o protocolo de treinamento em 4 indivíduos. Todos os participantes tinham em comum a hipertensão arterial sistêmica. Os 2 primeiros realizaram 16 
sessões, e os outros 2 realizaram apenas 8 sessões. Para aqueles que realizaram apenas 8 sessões foram aplicadas apenas 2 avaliações (Pré e Pós 8 ).

O participante P09, de 48 anos, do sexo masculino, sofreu o AVC no dia 17/07/2017 e iniciou as aplicações da interface EMG-FES no dia 22/08/2019 Como comorbidades, relatou diabetes mellitus (DM) e dislipidemia, sedentário, sem realizar fisioterapia. O participante realizou 16 sessões e quatro avaliações. Apresentou principalmente aumento do descritor RMS do músculo extensor do indicador, houve aumento do descritor MDF em todos os músculos avaliados. Evidenciou também redução da espasticidade no grupo muscular de flexão dos dedos.

O segundo participante, P10, de 56 anos, do sexo masculino sofreu o AVC isquêmico no dia 14/12/2018 e iniciou as aplicações da interface EMG-FES no dia 22/08/2019. Como comorbidades relatou DM, psoríase e bursite na articulação do ombro esquerdo. Já havia sofrido outro AVC. O participante realizou 16 sessões e 4 avaliações. Apresentou principalmente aumento dos descritores RMS e MDF no grupo muscular de extensão dos dedos, extensor do indicador e flexor dos dedos.

A terceiro participante, P13, de 38 anos, do sexo feminino sofreu o AVC isquêmico no dia 18/06/2014 e iniciou as aplicações da interface EMG-FES no dia 22/11/2019. Como comorbidades relatou depressão, não frequenta a fisioterapia e faz exercício físico 2 vezes na semana. A participante realizou 8 sessões e 2 avaliações. Apresentou 
aumento do descritor MDF dos músculos extensor do indicador e extensor dos dedos, houve também aumento do descritor RMS em todos os músculos avaliados, principalmente do grupo muscular extensor, evidenciou também redução da espasticidade do grupo muscular de flexão dos dedos.

O quarto participante, P14, de 58 anos, do sexo masculino sofreu o AVC hemorrágico no dia 17/06/2019 e iniciou as aplicações da interface EMG-FES no dia 13/01/2020. Como comorbidades relatou presença de artrose na articulação do quadril esquerdo, e realizava fisioterapia 2 vezes na semana. O participante realizou 8 sessões e 2 avaliações. Apresentou aumento do descritor MDF dos apenas do músculo abdutor de polegar, houve também aumento do descritor RMS nos músculos extensores de indicador, no grupo muscular de extensão e o de flexão dedos, com ênfase no grupo muscular de extensão.

A Figura 4 apresenta o sinal registrado pela EMG referente ao descritor RMS captado de cada músculo, relacionado a capacidade de força muscular destes músculos. Cada músculo avaliado foi plotado em um gráfico único, como indicado pelos títulos em vermelho. A distribuição dos dados de todos os participantes foi evidenciadas nos quatro momentos de coleta que está indicados no eixo horizontal de cada gráfico bloxplot. Os dados de Pre e Pós 8 referem-se às coletas realizadas em 4 participantes, enquanto, os Pós 16 e Pósfu foram coletas realizadas em 2 participantes. Já a Figura 5 representa o 
sinal registrado pela EMG referente ao descritor MDF captado de cada músculo, relacionado a taxa de disparo do motoneurônio. Os gráficos seguiram a mesma lógica de plotagem da Figura 4, alterando apenas 0 descritor analisado.

Figura 4. Gráficos analisam a raiz média quadrática do sinal (RMS).
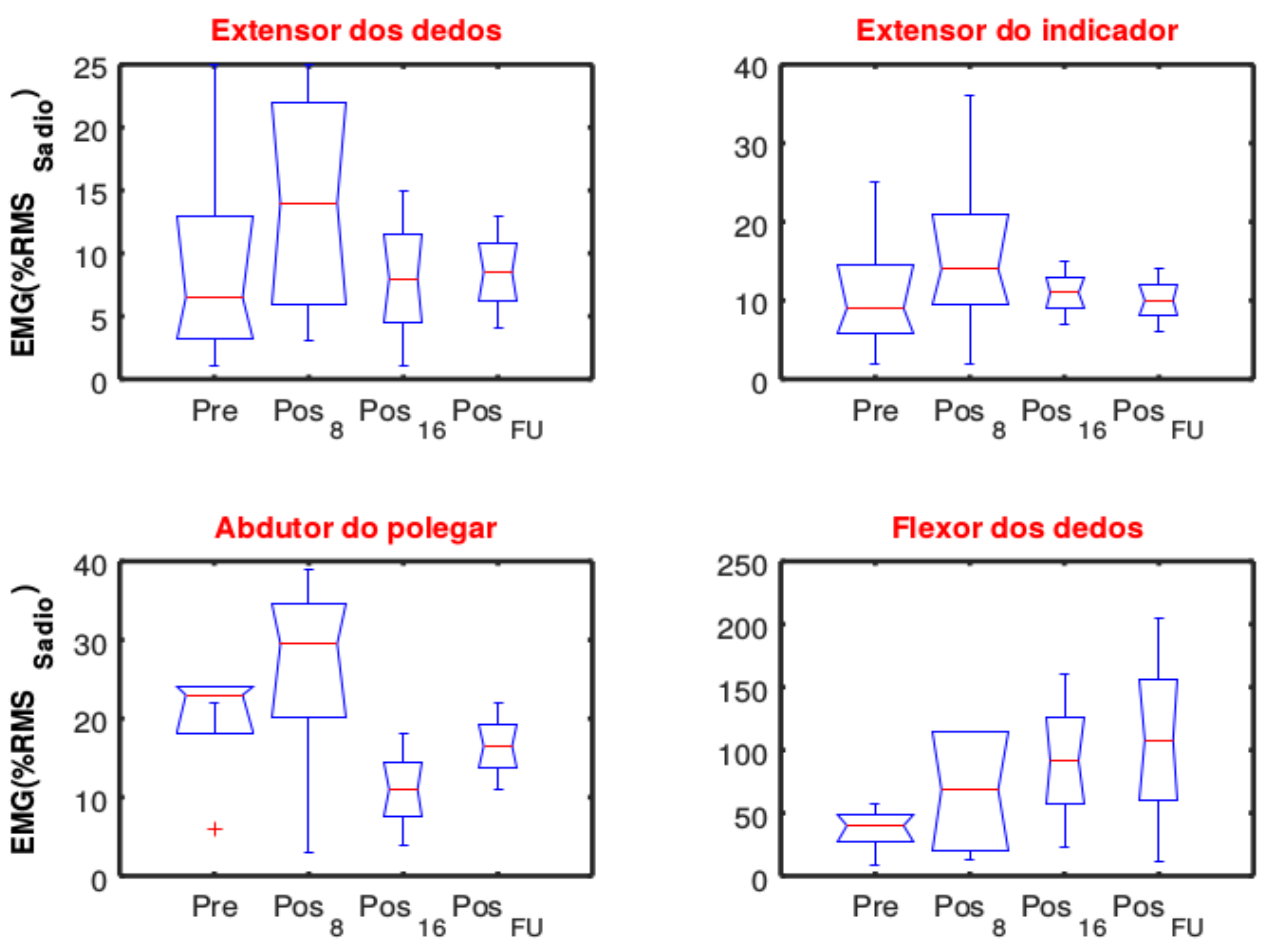
Figura 5. Gráficos analisam a frequência mediana do sinal (MDF).
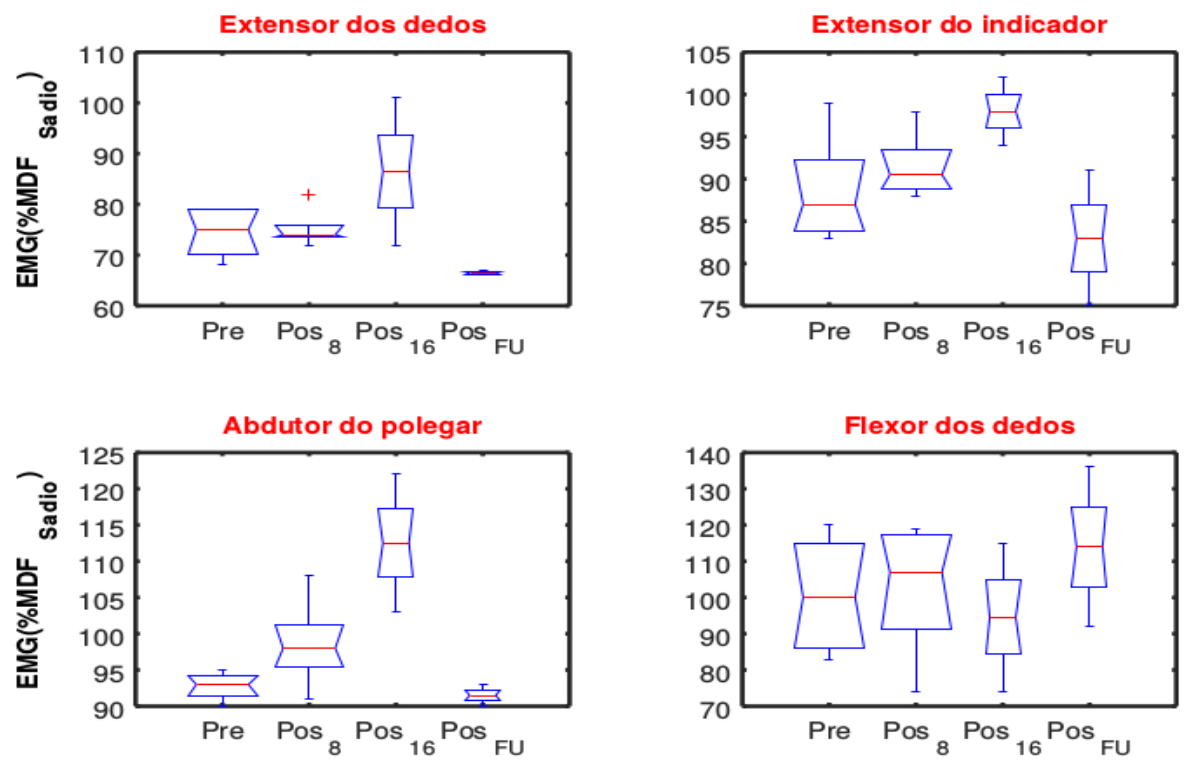

\section{Avaliação Pré}

Os participantes realizaram a avaliação Pré, com dados diferentes entre si, identificando que o sinal basal de cada um é particular, assim como seus déficits motores. Dois participantes foram submetidos a 40 e 80 horas da interface cérebro-máquina, utilizando a FES nos músculos extensores da mão ${ }^{8}$. Mostra que na primeira avaliação há possibilidade dos participantes que apresentaram pontuação maior no sinal basal podem ter melhor prognóstico. A FES está entre as poucas terapias mostradas até hoje que é eficaz em indivíduos com hemiplegia crônica, uma vez que se considera que a população não tem respondido à reabilitação convencional e a efetividade da FES ocorre pelas alterações neuroplásticas, que ocorrem devido à presença coordenada 
de um comando motor seguido pelo feedback sensorial correspondente ao movimento assistido por estimulação ${ }^{8}$.

\section{Avaliação Póss ( $\mathbf{N}=4)$}

$\mathrm{Na}$ avaliação Póss todos os grupos musculares avaliados obtiveram aumento referente à ambos os descritores, RMS e MDF. Quanto ao músculo abdutor do polegar hipotetiza-se que devido ao difícil posicionamento do eletrodo na superfície do músculo, acredita-se que não há fidelidade a avaliação. Em um relato de caso, com protocolo similar, foi aplicado a interface EMG-FES por 30 minutos em 12 sessões por 3 vezes, e houve aumento da funcionalidade e aumento dos descritores RMS e MDF do músculo extensor dos dedos. Foi necessário reduzir os parâmetros da Interface EMG-FES durante $\mathrm{o}$ período de tratamento, evidenciando a neuroplasticidade promovida pela interface ${ }^{9}$. Utilizando a FES em protocolo, associada a eletroencefalografia (EEG) ao invés da EMG, em um homem de 64 anos, foram aplicadas 40 sessões de 90 minutos no membro superior lesado após

6 anos do AVC. À medida que o participante recupera a função voluntária, o uso da FES diminui gradualmente até não ser necessário seu uso para realizar movimentos funcionais ${ }^{10}$. Esse estudo indica que mesmo com um participante acima de 60 anos de idade e com 6 anos após o AVC, ainda conseguiu-se induzir uma neuroplasticidade por meio de uma interface EEG-FES.

$\mathrm{Na}$ avaliação do grupo muscular flexor dos dedos houve aumento da RMS, relacionado a co-contração, evidenciando 
a dificuldade dos participantes em realizar apenas a contração do grupo muscular extensor dos dedos, porém durante as intervenções foi notada a quebra da espasticidade. Em uma revisão bibliográfica, observou-se que frequências da FES menores que $20 \mathrm{~Hz}$ causam contrações fasciculadas ineficazes para ganho de força, consequentemente ineficaz para promoção de movimento funcional ${ }^{4}$. No presente estudo, o uso da FES com frequência modulada iniciando em $5 \mathrm{~Hz}$ pode ter causado reflexo na musculatura antagonista ao grupo muscular extensor de dedos. Essa hipótese é evidenciada por meio do aumento dos descritores RMS e MDF no grupo muscular flexor de dedos. Desse modo, seria melhor iniciar a FES com frequências superiores a $20 \mathrm{~Hz}$, a fim de evitar contrações fasciculadas.

\section{Avaliação Pós $16(\mathbf{N}=2)$}

Na avaliação Pós16 o grupo muscular extensor e o músculo abdutor do polegar apresentaram diminuição do descritor RMS e apresentam aumento do descritor MDF ao comparar com a avaliação Pós8. O grupo muscular flexor dos dedos apresentou aumento do descritor RMS e o apresentou diminuição do descritor MDF. Durante 40 sessões de aplicação, a FES associada a EEG, foram realizadas três avaliações: antes, após 20 sessões e após 40 sessões. Assim como esse protocolo, realizou uma avaliação no ponto médio das sessões, na avaliação de independência funcional aumentou de 104/126 para 118/126 da avaliação antes da intervenção até a avaliação de ponto médio e na última 
avaliação manteve 118/126. No teste Fugl-Meyer para membro superior teve pontuação 13 antes na intervenção, 18 na avaliação de médio ponto e 19 na última avaliação. O teste de função da mão de Toronto (TRI-HFT) que envolvem manipulação de objetos, relacionado a função motora da mão com a atividade de vida diária, o qual manteve-se com valor zerado do início ao $\mathrm{fim}^{10}$. Em conformidade com 0 presente estudo, verificou possível estágio em que não há mais evolução na avaliação de ponto médio, assim, atingindo um possível "efeito teto" de melhoria. Em contraponto com o presente estudo, esse estudo teve enfoque no braço ao invés da mão, priorizando a melhora da função de alcance do braço e posteriormente buscou reabilitar as fases do movimento, iniciado por movimentos proximais evoluindo a distais ${ }^{10}$.

\section{Avaliação Pósfu $(\mathbf{N}=2)$}

$\mathrm{Na}$ avaliação de acompanhamento, pode-se notar que o grupo muscular extensor manteve os valores do RMS ao comparar-se com a avaliação Pós 16 . O descritor MDF na avaliação Pósfu apresentou diminuição, pois o grupo muscular extensor e o músculo abdutor do polegar apresentaram valores inferiores a avaliação Pré. Enquanto o grupo muscular flexor obteve valores maiores tanto em RMS quanto em MDF. Num ensaio clínico foi comparado o treinamento bilateral do ombro com uso da estimulação elétrica transcutânea ou FES. Randomizou-se em dois grupos 38 participantes pós AVC que realizaram 12 sessões durante 
4 semanas, avaliados antes, após 12 sessões e avaliação de acompanhamento (30 dias após 12 sessões). A avaliação de acompanhamento, do grupo tratado com FES, mostrou aumento: (i) do movimento do braço hemiparético, (ii) atenção cognitiva por meio da proprioceptividade feedback sensorial e (iii) melhora da função do braço ${ }^{11}$. A maior frequência semanal de terapia pode estar associada ao aumento de parâmetros a longo prazo, pois enquanto no estudo citado faziam 3 vezes por semana, no presente estudo eram feitas 2 vezes por semana.

O descritor RMS não obteve aumento nas avaliações Pós 16 e Pósfu, pois os dois primeiros participantes que realizaram exercícios de resistência durante a aplicação da interface EMG-FES, a resistência iria estimular o ganho de força muscular, aumentando 0 descritor RMS proporcionalmente o MDF. Em uma metanálise que comparou 30 estudos ( $\mathrm{N}=1.051)$ sobre treinamento resistido em pessoas com sequelas de AVC, verificou-se que são superiores a outras terapias quanto ao ganho de força muscular e funcionalidade, além de melhorar outros indicadores fisiológicos relevantes para saúde. Para otimizar o exercício resistido, os estudos mostram que é eficaz associá-los à de alta intensidade em exercícios concêntricos ou excêntricos. Quanto a espasticidade, o autor indica que o exercício resistido pode reduzi-la, porém os estudos ainda são limitados ${ }^{12}$.

Na avaliação Pósfu o grupo muscular flexor obteve valores maiores tanto em RMS quanto em MDF, enquanto os 
músculos antagonistas regrediram seus valores, evidenciando que não houve neuroplasticidade a longo prazo no grupo muscular extensor. Em uma revisão bibliográfica, foi observado que a neuroplasticidade a longo prazo é representada pela diminuição da ativação do músculo antagonista (espástico) 4 .

No presente estudo, sugere-se que a aplicação da interface EMG-FES poderia ser otimizada se fosse adicionada a terapia do espelho (TE), na qual combina os movimentos bilaterais com o uso do espelho entre os membros, de modo esconder o membro afetado e refletir o membro saudável, associa o treino sensório-motor com feedback visual simulando atividade normal bilateral, estimulando a melhoria funcional encefálica ${ }^{13}$. Em uma revisão bibliográfica com estudos randomizados controlados e/ou estudos piloto, que associavam a TE em membro superior em pacientes pós AVC, foi incluído 9 artigos, totalizando 230 participantes, e foi observada melhora da recuperação funcional, das atividades da vida diária e velocidade do movimento. Um dos artigos incluídos associava a TE com a FES em 20 participantes, mostrou melhoria na amplitude da extensão de punho ${ }^{13}$. Além do mais, em participantes que não possuem contração residual a terapia do espelho poderia ser associada com a interface EMG-FES, usando inicialmente a EMG no lado saudável e a FES no lado parético, para ganho de neuroplasticidade e contração do lado parético, para evoluir para a aplicação do protocolo deste estudo: uso da 
interface EMG-FES em um mesmo membro, em participantes que apresentem contração residual.

Em uma revisão de literatura, verificou-se em um estudo randomizado sobre uso assistência robótica em pessoas que sofreram AVC mostrou que esta terapia com assistida não é melhor que os cuidados oferecidos pela reabilitação convencional. Uma vez que a reabilitação é crucial no fornecimento de estímulos necessário para recuperação quando aplicada imediatamente após o início da plasticidade ao invés de concomitante com a recuperação ${ }^{14}$.

\section{CONCLUSÃO}

O uso interface EMG-FES, em 8 a 16 sessões com duração de aproximadamente 30 minutos cada em 4 pessoas pós AVC crônico, se mostrou eficiente na avaliação de eletromiografia de superfície, com o aumento dos descritores RMS e MDF dos músculos extensores de punho e dedos apenas a curto prazo.

Para trabalhos futuros pode-se aplicar um sistema de controle em malha fechada com correção automática dos parâmetros da FES, associação de terapia do espelho, bem como progressão da conduta adicionar o treino resistido. Para continuidade deste estudo a questão de um possível "efeito teto" deve ser mais bem avaliada para otimizar a neuroplasticidade, garantindo efeitos a longo prazo. 


\section{REFERÊNCIAS}

1.Delboni MCC, Malengo PCM, Schmidt EPR. Relação entre os aspectos das alterações funcionais e seu impacto na qualidade de vida das pessoas com sequelas de Acidente Vascular Encefálico (AVC). O Mundo da Saúde 2010;35:165-75. https://doi.org/10.15343/01047809.20102165175

2.Balasubramanian S, Garcia-Cossio E, Birbaumer N, Burdet E, RamosMurguialday A. Is EMG a Viable Alternative to BCI for Detecting Movement Intention in Severe Stroke? IEEE Trans Biomed Eng 2018;65:2790-7. https://doi.org/10.1109/tbme.2018.2817688

3.Ambrosini E, Ferrante S, Zajc J, Bulgheroni M, Baccinelli W, d'Amico $\mathrm{E}$, et al. The combined action of a passive exoskeleton and an EMGcontrolled neuroprosthesis for upper limb stroke rehabilitation: first results of the RETRAINER project. International Conference on Rehabilitation Robotics (ICORR) 2017, p. 56-61. https://doi.org/10.1109/icorr.2017.8009221

4.Krueger-Beck E, Scheeren EM, Neto GNN, Button VLSN, Nohama P. Efeitos da estimulação elétrica funcional no controle neuromuscular artificial. Rev Neurocienc 2011;19:530-41. https://doi.org/10.4181/rnc.2010.06ip.11

5.Camona C, Wilkins KB, Drogos J, Sullivan JE, Dewald JPA, Yao J. Improving hand function of severely impaired chronic hemiparetic stroke individuals using task-specific training with the rein-hand system: a case series. Front Neurol 2018;9:923. https://doi.org/10.3389/fneur.2018.00923

6.Bae S, Kim KY. Dual-afferent sensory input training for voluntary movement after stroke: a pilot randomized controlled study. NeuroRehabil 2017;40:293-300. https://doi.org/10.3233/nre-161417 7.Persch AC, Gugiu PC, Velozo CA, Page SJ. Rasch analysis of the wrist and hand fugl-meyer. J Neurol Phys Ther 2015;39:185-92. https://doi.org/10.1097/npt.0000000000000096

8.Jovanovic LI, Desai N, Marquez-Chin C. Restoration of upper limb voluntary motor function in chronic severe hemiplegia using a braincomputer interface-triggered functional electrical stimulation therapy. IEEE International Conference on Systems, Man and Cybernetics (SMC) 2019, Bari, p.1292-7. https://doi.org/10.1109/smc.2019.8914024 9. Fiusa JM, Yonamine GS, Fumagali GLC, Moraes GF, Nohama P, Krueger E. The Muscle-Machine Interface After Stroke in Improvement of Hand Extension: Case Report. Commun Comput Inf Sci 2019;1068:249-57. https://doi.org/10.1007/978-3-030-36636-018 10. Marquez-Chin C, Marquis A, Popovic MR. EEG-Triggered Functional Electrical Stimulation Therapy for Restoring Upper Limb Function in Chronic Stroke with Severe Hemiplegia. Case Rep Neurol Med 2016;2016:1-11. http://dx.doi.org/10.1155/2016/9146213

11.Chuang LL, Chen YL, Chen CC, Li YC, Wong AMK, Hsu AL, et al. Effect of EMG-triggered neuromuscular electrical stimulation with bilateral arm training on hemiplegic shoulder pain and arm function after stroke: A randomized controlled trial. J Neuroeng Rehabil 2017;14:1-12. https://doi.org/10.1186/s12984-017-0332-0 
12.Veldema J, Jansen P. Resistance training in stroke rehabilitation: systematic review and meta-analysis. Clin Rehabil 2020;34:1173-97. https://doi.org/10.1177/0269215520932964

13.Magalhães CRM, Vasco A. Efeito da Terapia de Espelho na reabilitação de pacientes com sequelas de Acidente Vascular Encefálico: Uma revisão bibliográfica (Tese). Porto: Universidade Fernando Pessoa, 2017. http://hdl.handle.net/10284/6288

14. Nagappan PG, Chen H, Wang DY. Neuroregeneration and plasticity: a review of the physiological mechanisms for achieving functional recovery postinjury. Mil Med Res 2020;7:30. https://doi.org/10.1186/s40779-020-00259-3 